\title{
Detection of carotid occlusive disease by pulsed Doppler spectral analysis
}

\author{
D RUSSELL, K-F LINDEGAARD, P NAKSTAD, R NYBERG-HANSEN, KG ØYGARDEN \\ From the Departments of Neurology, Neurosurgery, and Radiology, Section of Neuroradiology, \\ Rikshospitalet, The National Hospital, University of Oslo, Oslo, Norway
}

SUMMARY In this study a new pulsed Doppler spectral analysis system was used for the evaluation of extracranial carotid disease. When compared to the arteriography findings in 224 carotid arteries, the Doppler examination correctly detected all stenoses greater than $25 \%$, all occlusions, all normal vessels, and $43 \%$ of stenosis causing a diameter reduction of less than $25 \%$.

Doppler ultrasound is now an accepted method for the clinical detection and evaluation of extracranial carotid disease. Problems, however, still exist in the assessment of minor stenoses which are not haemodynamically significant and severe stenosis or occlusion of the internal carotid artery. ${ }^{1}$ In an attempt to improve this examination, assessment of the flow pattern with spectral analysis of the Doppler signal, using both continuous ${ }^{2-5}$ and pulsed Doppler systems, ${ }^{6-11}$ has provided promising results. It is still undecided, however, which of these two systems is the more suitable for studying flow patterns in the clinical situation. ${ }^{12}$ We therefore present our experience with a newly developed pulsed Doppler spectral analysis system in the evaluation of extracranial carotid disease.

\section{Materials and methods}

\section{Instrumentation}

The pulsed Doppler apparatus (Vingmed Inc., Oslo, Norway) has four frequencies, $1,2,5$ and $10 \mathrm{MHz}$. The size of the sample volume can be selected in two different modes, high or low resolution. Using $5 \mathrm{MHz}$ and low resolution the size of the sample volume can be approximated to a cylinder with diameter and length of $4 \mathrm{~mm}$. With $5 \mathrm{MHz}$ and high resolution, the cylindrical sample volume has an approximate diameter of $4 \mathrm{~mm}$ and length of $2 \mathrm{~mm}$. This system also contains a variable high-pass filter which can remove Doppler shifts caused by movement of the vessel wall. In this study the filter was set to a cut-off frequency of

Address for reprint requests: David Russell, MD Department of Neurology, Rikshospitalet, The National Hospital, University of Oslo, Oslo 1, Norway.

Received 30 October 1983 and in revised form 22 January 1984. Accepted 30 January 1984
$100 \mathrm{~Hz}$ corresponding to a velocity of $0,015 \mathrm{~m} / \mathrm{s}$, using 5 $\mathrm{MHz}$ transmitted frequency. A digital display on the Doppler system shows the temporal peak value of the maximum velocity or the time average of the spatial mean velocity within the sample volume. In addition, analogue outputs for the maximum and the mean velocities are displayed on a monitor. The spectral analyser (Vingmed Inc.) provides a Fourier analysis of the Doppler signal based on the chirp-Z-transform.

The Doppler spectrum, together with ECG, and maximum and mean velocities were displayed in real-time on a monitor and could be written out directly on a fiberoptic recorder, the Doppler spectrum being presented as a grey-scale printout. The Doppler spectrum was also recorded with $40 \mathrm{KHz}$ bandwidth on a specially modified stereo tape recorder together with comments made by the examiner. This system enabled the recording of the frequency range available using the spectral analysis system.

Pulsed Doppler systems are limited as regards the maximum frequency which can be detected. This arises from Shannon's sampling theorem. The highest detectable frequency is half the pulse repetition rate of the Doppler, and is known as the Nyquist frequency. The Doppler system used in this study has a variable pulse repetition rate allowing for the highest possible Nyquist's frequency at the selected depth. Spectral analysis of the complex Doppler signal enables measurement of frequencies up to twice Nyquist's frequency when blood is moving in the same direction. ${ }^{13}$

The findings presented in this study are based on $5 \mathrm{MHz}$ pulsed frequency and low resolution mode.

\section{Doppler examination and evaluation}

The common carotid artery was first identified low in the neck just above the clavicle. The probe was then moved slowly downstream along the common (CCA) and internal carotid arteries (ICA) at an angle approximately $45^{\circ}$ to the vessel.

Blood velocities vary across the artery lumen because of viscous friction between the blood and vessel wall. Blood 
velocity is therefore approximately zero at the wall and largest at the centre of the vessel..$^{14}$ Acceleration of the blood when the heart is pumping also effects the velocity distribution in the artery by flattening the profile. The distribution of velocities depends therefore on both the viscosity and the acceleration of the blood. In the CCA and ICA there is normally substantial diastolic flow. The velocity profile is then nearly parabolic. In the acceleration phase of systole, the profile is flatter and in the retardation phase in late systole there can even be negative velocities close to the artery wall because the retardation adds a flat negative component to the profile. ${ }^{14}$ The Doppler spectrum from the CCA and ICA therefore normally shows in systole and especially in the acceleration phase, a narrow band in the upper part of the spectrum where velocities are concentrated with a clear area beneath (fig $1, a, b)$.

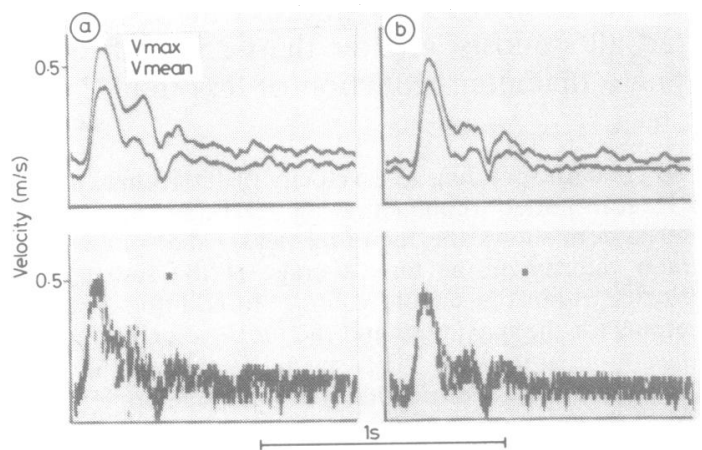

Fig 1 Doppler spectra recorded from arteriographically normal CCA (a) and ICA (b) Top: Vmax and Vmean, bottom: Velocity spectrum.

In assessing the Doppler findings particular attention was paid to the Doppler spectrum in the acceleration phase of systole. A lesion was diagnosed when the Doppler spectrum in a localised area of the vessel under study changed such that frequencies could clearly be seen in both the upper and lower parts of the spectrum in the acceleration phase of systole. Relative changes in the Doppler spectrum in the decelleration phase of systole, or in diastole, were not assessed as being due to a lesion of the vessel. When maximum velocity at the site of the spectral spreading was not increased by more than $10 \%$, compared to the maximum velocity downstream in the ICA, at the angle of the jaw, a diagnosis of stenosis less than $10 \%$ (that is wall irregularities) was made. When the maximum velocity at the site of the spectral spreading $\left(\mathrm{V}_{1}\right)$ was increased by more than $10 \%$, compared to the maximum velocity downstream in the ICA at the angle of the jaw $\left(V_{2}\right)$, the percentage stenosis was calculated as follows: Stenosis (Doppler) $=\left(1-\mathrm{V}_{2} / \mathrm{V}_{1}\right) \times 100 \%$. An increase in maximum velocity alone without spectral spreading in the acceleration phase of systole was not assessed as being due to a lesion of the vessel.

The Doppler studies were carried out by one of us (DR) without knowledge of the arteriography results.

\section{Patients}

During an 18 month period, 420 patients with symptoms suggestive of cerebrovascular disease were examined. On the basis of their history and clinical findings $112(27 \%)$ patients were also examined by arteriography. Arteriographic films of 224 carotid arteries were therefore available for comparison with the results of the Doppler examinations. These are the subject of the present study.

Biplanar arteriography of both extracranial and intracranial arteries was obtained by the transfemoral route. The arteriographic films were interpreted from a single plane view which showed the most severe abnormality. The degree of stenosis was based on the percentage diameter reduction at the area of the most severe stenosis relative to the diameter of the distal internal carotid artery. When there was stenosis of the common carotid artery, the percentage diameter reduction was related to the normal common carotid diameter. Calipers were used to measure the diameter in the stenotic and non-stenotic zones. All arteriograms were interpreted independently by two observers who were unaware of the results of the Doppler examination.

\section{Results}

The results of the Doppler and arteriography examinations are shown in the table. All 131 arteriographically normal vessels were assessed correctly using the Doppler system. Ninety-three $(42 \%)$ of the 224 vessels examined showed lesions on arteriography; these ranged from minor wall irregularities to occlusion of the internal carotid artery. Seventy-six $(82 \%)$ of these arteriographically abnormal vessels were also assessed as having a lesion on Doppler examination. All 46 stenoses causing a diameter reduction more than $25 \%$ on arteriography were found by Doppler examination

Table A comparison of the Doppler and arteriography findings. $\%=\%$ diameter reduction

\begin{tabular}{|c|c|c|c|c|c|c|c|c|}
\hline \multirow{2}{*}{$\begin{array}{l}\text { Arteriography } \\
\text { findings }\end{array}$} & & \multicolumn{7}{|c|}{ Doppler finding } \\
\hline & & Normal & $<10 \%$ & $11-25 \%$ & $26-50 \%$ & $51-75 \%$ & $76-99 \%$ & Occlusion \\
\hline $\begin{array}{l}\text { Normal } \\
<10 \% \\
11-25 \% \\
26-50 \% \\
51-75 \% \\
76-99 \% \\
\text { Occlusion }\end{array}$ & $\begin{array}{r}131 \\
23 \\
7 \\
13 \\
17 \\
16 \\
17\end{array}$ & $\begin{array}{r}131 \\
14 \\
3\end{array}$ & 9 & $\begin{array}{l}2 \\
1\end{array}$ & $\begin{array}{l}1 \\
5 \\
3\end{array}$ & $\begin{array}{l}1 \\
6 \\
6 \\
7\end{array}$ & $\begin{array}{l}1 \\
8 \\
9\end{array}$ & 17 \\
\hline
\end{tabular}



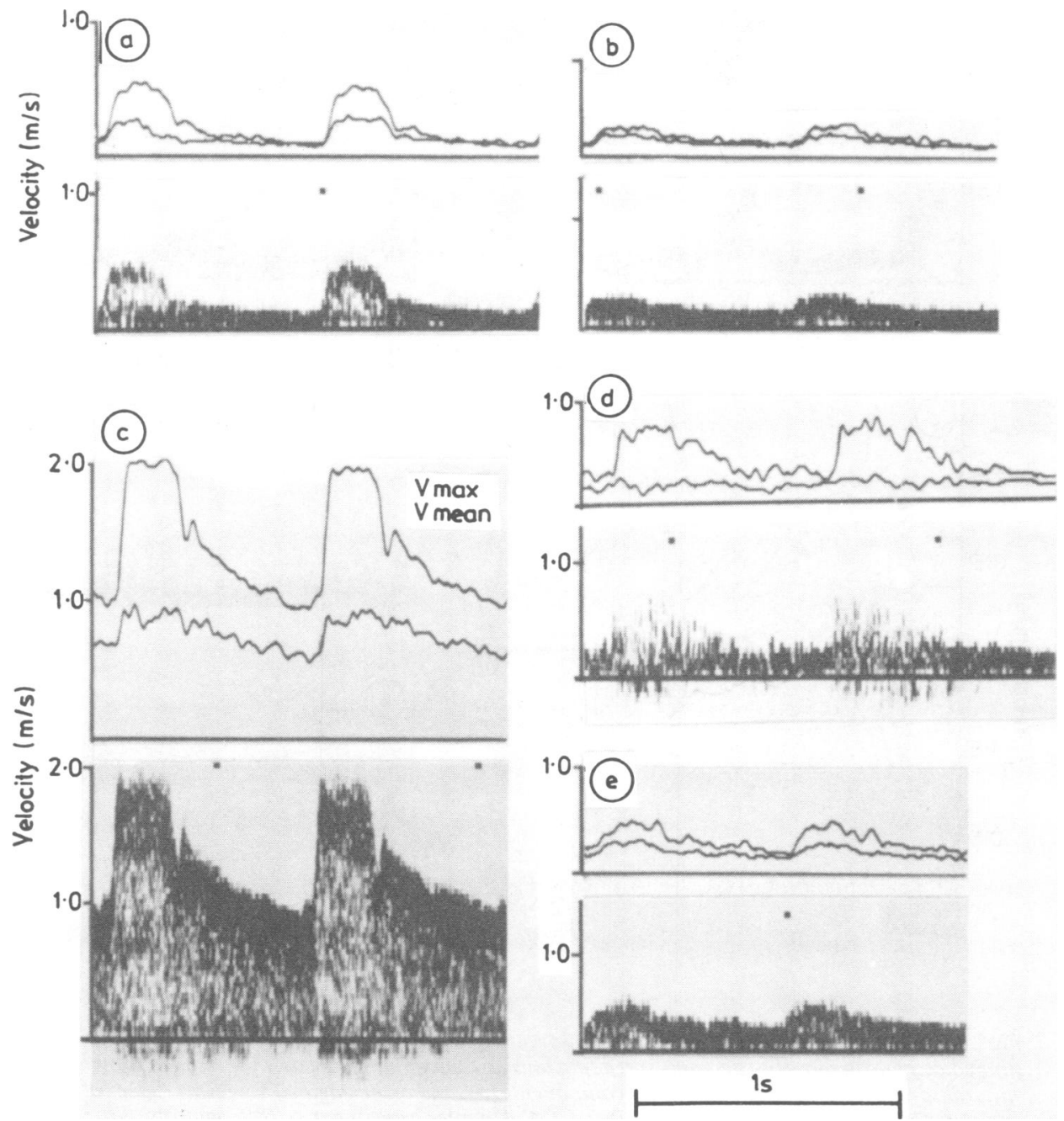

Fig 2 Doppler findings at the site of a stenosis of an ICA (c), with spectral spreading and high maximum velocities $(2.0 \mathrm{~m} / \mathrm{s})$. Upstream: spectral spreading in the CCA (a), and decrease in the maximum velocity $(0.23 \mathrm{~m} / \mathrm{s})$ immediately upstream (b) to the stenosis. Downstream: spectral spreading and negative velocity components $(d)$, and at the angle of the jaw (e) spectral spreading is still present and the maximum velocity is $0.37 \mathrm{~m} / \mathrm{s}$. Doppler assessment $=81 \%$ stenosis. Arteriography $=70 \%$ diameter reduction.

(sensitivity $=100 \%)$. Disturbances in the Doppler spectrum were also frequently seen both upstream and downstream to stenoses, where there was a diameter reduction of greater than $50 \%$ (figs 2,3 ). In the CCA spectral spreading, and immediately upstream a reduction in maximum velocity were recorded, whereas spectral spreading and negative velocity components were usually present for a variable length downstream to these stenoses.

In seven vessels the arteriography findings were assessed as showing a $11-25 \%$ stenosis. Doppler examination detected four of these lesions $(57 \%)$.

Nine $(39 \%)$ of the 23 vessels in which wall irregularities (that is diameter reduction less than $10 \%$ ) were seen on arteriography were also diagnosed as having a lesion on Doppler examination. Figure 4 shows the Doppler findings in one of these vessels. In a localised area of the ICA spreading of the Doppler spectrum was found in the acceleration phase of systole (fig 4c); however, the maximum 

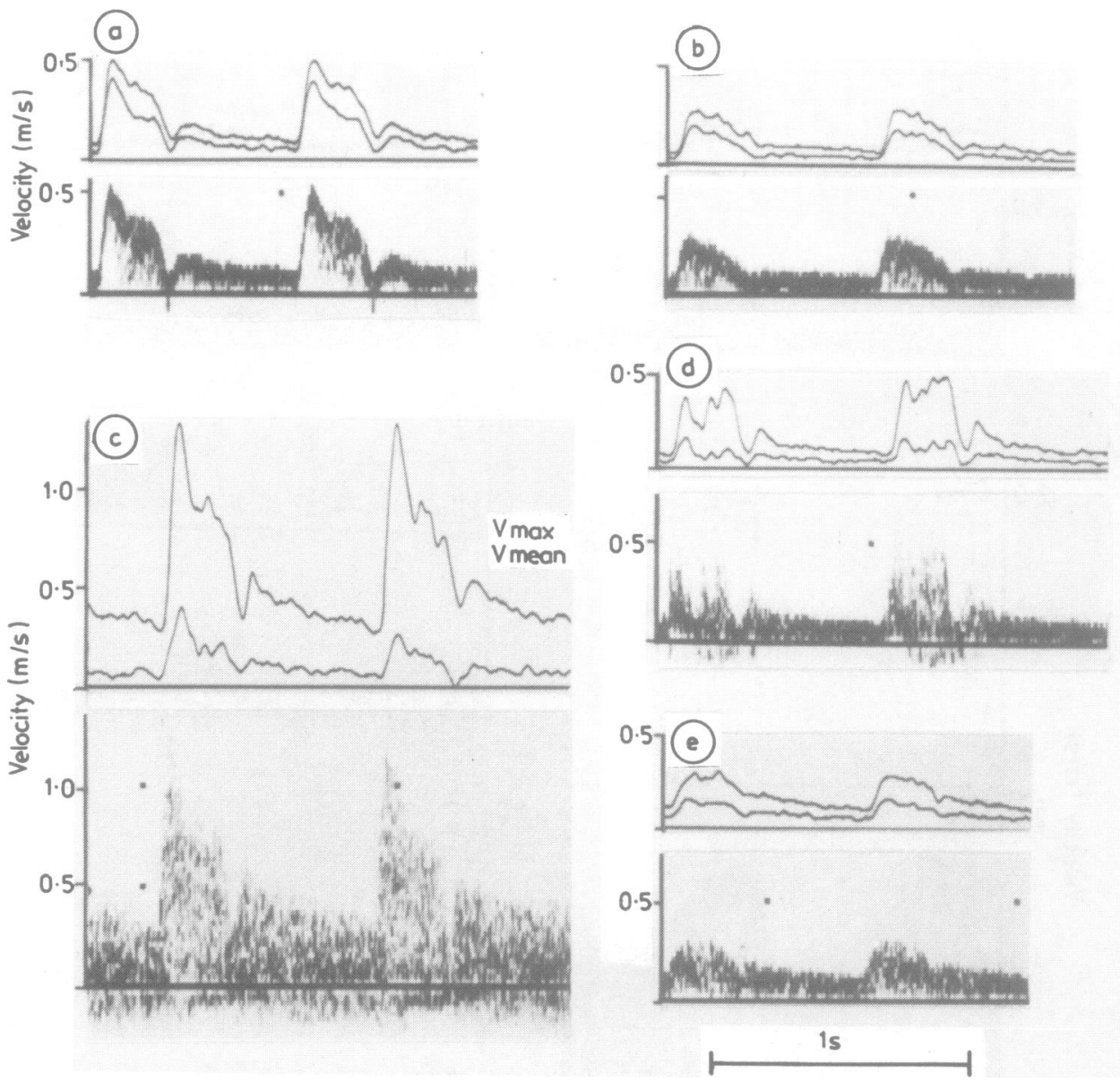

Fig 3 Stenosis of an ICA (C), with spectral spreading and high maximum velocities $(1.32 \mathrm{~m} / \mathrm{s})$. Immediately upstream (b) spectrum spreading and low maximum velocities $(0.27 \mathrm{~m} / \mathrm{s})$. Downstream: spectral spreading and negative velocity components $(d)$, and at the angle of the jaw (c) spectral spreading and a maximum velocity of $0.29 \mathrm{~m} / \mathrm{s}$. $(a)=C C A$. Doppler assessment $=78 \%$ stenosis. Arteriography $=$ $90 \%$ diameter reduction.

velocity $(0.58 \mathrm{~m} / \mathrm{s})$ was similar to that measured downstream at the angle of the jaw $(0.56 \mathrm{~m} / \mathrm{s}$, fig $4 d)$.

As can be seen from the table and figs 2 and 3, assessment of the percentage stenosis on the basis of maximum velocity increase tended to give higher values when compared to arteriography for stenoses from 25 to $75 \%$, whereas for stenoses from 75 to $99 \%$ the tendency was to underestimate the severity stenosis.

All 17 occluded ICAs were correctly diagnosed by Doppler examination. In these patients bifurcation of the CCA into the ICA and ECA was not found. However, if the ICA is occluded, the Doppler spec- trum from the ECA may resemble that of the ICA, due to increased flow in the ECA and changes in its peripheral resistance. It can therefore be difficult to decide whether the remaining vessel is the ECA or the ICA. The following test, however, was positive in all 17 cases of occlusion of the ICA. The Doppler probe is moved as far cranially as possible on the remaining vessel (that is ICA or ECA) and the Doppler signal observed continuously on the monitor and recorded. The ipsilateral temporal artery (or together with the facial artery) is then quickly compressed and released during the latter part of diastole. A short peak in the Doppler signal following this manoeuvre signifies that the vessel 

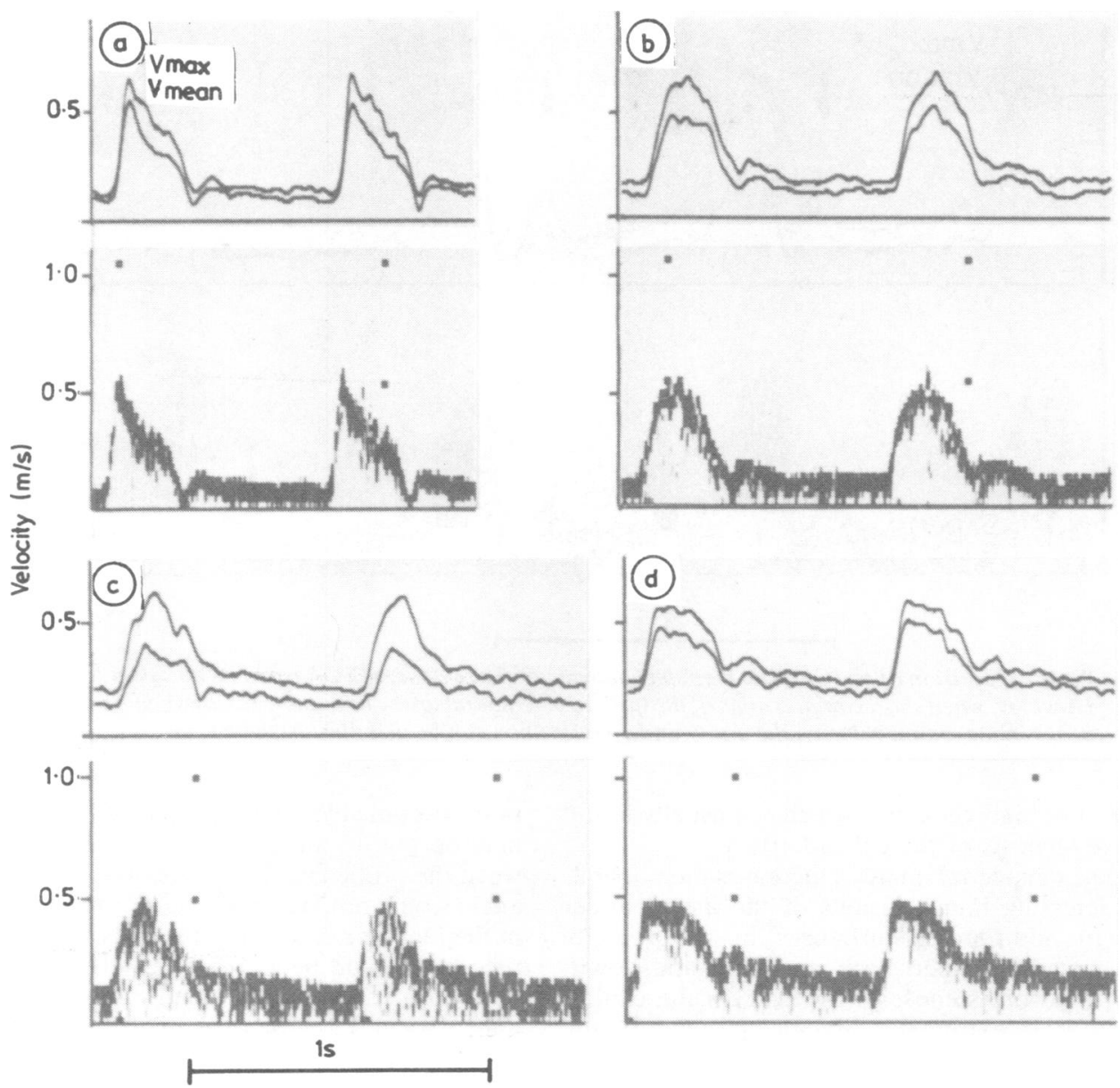

Fig 4 Doppler findings from an ICA where a localised area with spectral spreading in the acceleration phase of systole was found (c). This spectral spreading was not present in the CCA (a), immediately upstream (b), or downstream at the angle of the jaw (d). The maximum velocity at $(c)$ was similar to that measured downstream at $(d)$.

Doppler assessment $=<10 \%$ stenosis: arteriography $=$ wall irregularities.

which is under Doppler registration is the ECA. A diagnosis of occlusion of the ICA can then be made. Figure 5 shows the Doppler findings in one patient with occlusion of an ICA.

\section{Discussion}

In this study, the use of a new pulsed Doppler spectral analysis system enabled the detection of all stenoses causing a diameter reduction of more than $25 \%$ and all occlusions of the internal carotid arteries. Minor wall irregularities and stenoses causing a diameter reduction of less than $25 \%$ were detected in $43 \%$ of the cases. In addition all normal vessels were correctly identified. These results therefore represent an increased accuracy in the detection of carotid occlusive disease by Doppler examination. $^{2-11}$

The main advantage of pulsed Doppler over continuous wave systems in the study of velocity profiles is range resolution which prevents contamination of the velocity signal by unwanted signals from other vessels and reflected signals from the coupling gel. This allows for better control of the quality of the registration from the vessel under study. One disadvantage is that the maximal velocity which can be measured by pulsed systems is limited. The instrumetation used in this study, however, can 


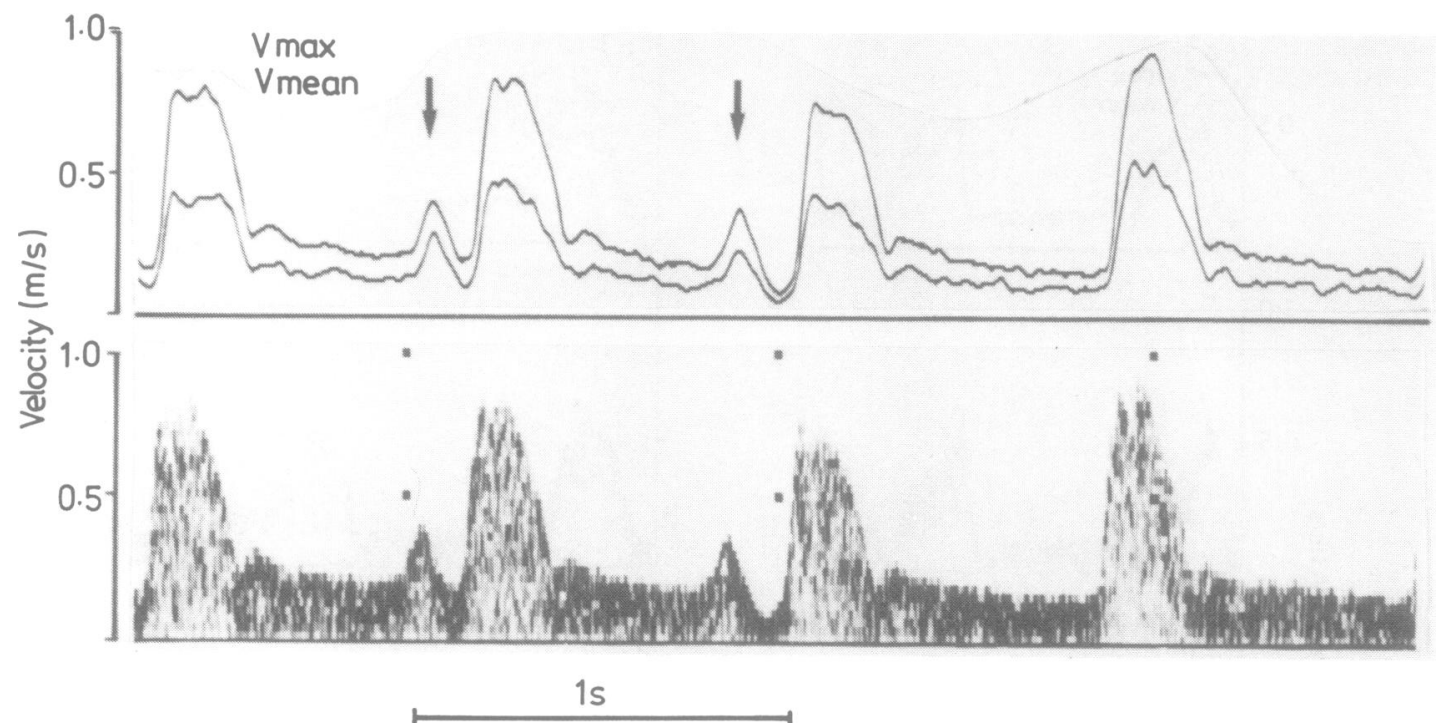

Fig 5 Occlusion of an ICA. The Doppler spectrum of the remaining ECA could be mistaken for an ICA. However, when short compressions of the ipsilateral temporal artery in diastole (arrows) give rise to short peaks in maximum velocity, the artery under registration can be identified as the ECA.

measure the high velocities which are usually found in severe stenosis of the carotid artery.

The use of spectral analysis increases the possibility of detecting minor lesions of the artery under study. In addition, disturbances in the velocity profile may be studied both upstream and downstream to severe stenoses. However, in the clinical situation it is extremely difficult to quantify the amount of spreading in the Doppler spectrum. Since the velocity profile in the CCA and ICA is nearly parabolic in diastole, spectral spreading is frequently present in this phase of the cardiac cycle. In the acceleration phase of systole the velocity profile is flatter and spectral spreading in this phase of the cardiac cycle is therefore more likely to be due to a lesion of the artery. In this study we assessed changes in the Doppler spectrum as being due to a lesion only when the Doppler spectrum changed so that frequencies were clearly seen in both the upper and lower parts of the spectrum in the acceleration phase of systole. These criteria also proved to be extremely helpful in the detection of lesions contralateral to a high grade stenosis or occlusion of the internal carotid artery, when the Doppler signal may be difficult to assess due to a compensatory increase in blood flow.

Spectral analysis is also helpful in assessing increases in maximum velocity, which may be found when the probe is passed over the carotid bifurcation onto the origin of the ICA. A relative increase in maximum velocity in this part of the examination may be due to an abrupt change in the angle between the probe and the axis of flow. A change in this angle would not, however, cause a relative spreading in the Doppler spectrum, since all of the frequencies recorded would be increased by the same factor.

In this study the Doppler sample volume was approximately cylindrical with a diameter and length of $4 \mathrm{~mm}$. This sample volume therefore usually provided a study of the velocity profile across a relatively large part of the lumen of the vessel under study. It is possible that the accuracy of this examination in the detection of stenoses less than $25 \%$ may be improved by using a smaller sample volume taken from the centre of the vessel. However, the significance of spectral spreading is difficult to assess when the sample volume is small compared to the ultrasound frequency, due to transit-time effects. ${ }^{12}$

Accurate results by Doppler examination were found in high grade stenosis and occlusion of the internal carotid artery, which suggests that this instrumentation is capable of detecting the weak Doppler signals which are present when flow is substantially reduced due to a high grade stenosis. Diagnosis of occlusion of an internal carotid artery may present problems using Doppler. We therefore wish to stress that short compressions of the ipsilateral temporal artery (or together with the facial artery) in late diastole, are extremely helpful in deciding whether the remaining artery is the ICA or 
the ECA. This differentiation has important practical consequences since occlusion of the internal carotid artery excludes the possibility of endarterectomy.

The results of this study show that pulsed Doppler spectral analysis can accurately assess normal vessels and those with a stenosis greater than $25 \%$. This examination may therefore be particularly helpful in the evaluation of the relatively large patient group who present with symptoms suggestive but not typical of extracranial carotid occlusive disease. In this patient group it is often difficult to assess the risks associated with arteriography against the possible therapeutic consequences. Doppler examination is also of special value in patients who have an increased risk of complication with arteriography, in those patients with asymptomatic bruits, and in situations where repeated examinations are desirable. However, in patients with typical symptoms of transient ischaemic attacks, a normal Doppler examination does not exclude the need for arteriography since small lesions not detected by the Doppler examination may be responsible for the patient's symptoms.

In the future, intravenous digital subtraction angiography may prove to be superior to Doppler in the assessment of minor lesions in the carotid vasculature. ${ }^{1516}$ This may suggest that studies using Doppler should now be directed towards a better assessment of the clinical significance of the haemodynamic information which may be measured by this method, rather than the detection of minor morphological changes.

\section{References}

' Zwiebel WJ, Crummy AB. Sources of error in Doppler diagnosis of carotid occlusive disease. AJR 1981;137:1-12.

${ }^{2}$ Reneman RS, Spencer MP. Local Doppler audio spectra and stenosed carotid arteries in man. Ultrasound Med Biol 1979; 5:1-11.
${ }^{3}$ Johnston KW, deMorais D, Kassam M, Brown PM. Cerebrovascular Assessment using a Doppler carotid scanner and real-time frequency analysis. J Clin Ultrasound 1981;9:443-9.

${ }^{4}$ Barnes RW, Rittgers SE, Putney WW. Real-time Doppler spectrum analysis Arch Surg 1982;117:52-7.

5 Johnston KW, Brown PM, Kassam M. Problems of carotid Doppler scanning which can be overcome by using frequency Analysis. Stroke 1982;13:660-6.

- Felix WR, Sigel B, Gibson RJ, et al. Pulsed Doppler ultrasound detection of flow disturbances in arteriosclerosis. J Clin Ultrasound 1977;4:275-82.

' Hobson RW, Benny SM, Katocs AS, O'Donnell JA, Jamil Z, Savitsky JP. Comparison of pulsed Doppler and real-time B-mode echo arteriography for noninvasive imaging of the extracranial carotid arteries. Surgery 1980;87:286-93.

${ }^{8}$ Blackshear WM, Phillips DJ, Thiele BL et al. Detection of carotid occlusive disease by ultrasonic imaging and pulsed Doppler spectrum analysis. Surgery 1979;86:698-706.

9 Blackshear WM, Phillips DJ, Chikos PM, Harley JD, Thiele BL, Strandness Jr. DE. Carotid artery velocity patterns in normal and stenotic vessels. Stroke 1980;64:67-71.

${ }^{10}$ Fell G, Phillips DJ, Chikos PM, Harley JD, Thiele BL, Strandness Jr. DE. Ultrasonic duplex scanning for disease of the caroid artery. Circulation 1981;64:1191-5.

"Keagy BA, Pharr WF, Thomas D, Bowes DE. A quantitative method for the evaluation of spectral analysis patterns in carotid artery stenosis. Ultrasound Med Biol 1982;8:625-30.

12 Reneman RS, Hoeks APG. Doppler Ultrasound in the Diagnosis of Cerebrovascular Disease. Chichester: Research Studies Press, 1982.

${ }^{13}$ Hatle L, Angelsen B. Doppler Ultrasound in Cardiology. Philadelphia: Lea and Febiger, 1982.

${ }^{14}$ McDonald DA. Blood flow in arteries. London: Edward Arnold, 1974

is Strother CM, Crummy AB. Cervical arteriosclerosisdiagnostic advances in need of a clinical answer. Stroke 1982;13:551-6.

${ }^{16}$ Acher CW, Turnipseed WD, Sackett JF, Strother CM, Crummy A, Mistretta C. Digital subtraction angiography and continuous-wave Doppler studies. Arch Surg 1983; 118:462-4. 